For floating point arithmetic

$$
\left|\frac{\epsilon(\operatorname{Arctan} x)}{\operatorname{Arctan} x}\right|<1.25 \cdot 10^{-20},
$$

and hence the calculated value differs from the true value by at most two units in the twentieth significant digit.

C. Errors in Arcsin $x$ and Arccos $x$.

The error for floating point arithmetic using twenty-one significant digits will be given. Arcsin $x$ will be calculated by means of

$$
\operatorname{Arcsin} x=\operatorname{Arctan} \frac{x}{\sqrt{1-x^{2}}}
$$

The quantity $1-x^{2}$ is calculated by means of $1-x^{2}=(1-x)(1+x)$. Then

$$
\left|\frac{\epsilon(\operatorname{Arctan} x)}{\operatorname{Arcsin} x}\right|<10^{-19},
$$

and Arcsin $x$ will be correct to within one unit in the nineteenth significant figure.

The error for Arccos $x$ is similar except that a round-off error due to subtraction is introduced. This error does not affect the conclusion that $\operatorname{Arccos} x$ will have been obtained correctly to within one unit in the nineteenth significant figure.

5. Conclusions. From the standpoint of machine application the procedure given is economical and yields precise results. It uses only twenty stored constants; the calculation of Arctan $x$ requires a maximum of only eleven multiplications and one division; the calculation of $\operatorname{Arcsin} x$ and $\operatorname{Arccos} x$ requires an additional multiplication, division, and square root.

Rich Electronic Computer Center

Engineering Experiment Station

Georgia Institute of Technology

Atlanta, Georgia

1. E. G. Kogbethinatz, "Computation of $\operatorname{Arctan} N$ for $-\infty<N<\infty$ using an electronic computer," IBM Jn. Res. and Dev., v. 2, 1958, p. 43-53.

2. E. G. KogBetLiantz, "Computation of Arcsin $N$ for $0<N<1$ using an electronic computer," IBM Jn. Res. and Dev., v. 2, 1958, p. 218-222.

3. Y. L. LuKe, "On the computation of $\log Z$ and Arctan $Z$," $M T A C$, v. 11, 1957, p. 16-18.

4. A. S. Housenolder, Principles of Numerical Analysis, McGraw Hill Book Company, New York, 1953.

\title{
The Calculation of Toroidal Harmonics
}

\section{By A. Rotenberg}

1. Introduction. It is the purpose of this note to describe the mathematical techniques employed in a code [5] for the IBM 704 to calculate toroidal harmonics (associated Legendre functions of half-integral order). We use recurrence techniques similar to those used by Goldstein and Thaler [1] in calculating Bessel func-

Received October 27, 1959. 
tions. The functions of the first kind, $P_{n-\frac{3}{3}}^{m}(x)$, and the second kind, $Q_{n-\frac{1}{2}}^{m}(x)$, both obey the recurrence relation

$$
(\nu-m) R_{\nu}{ }^{m}(x)=(2 \nu-1) x R_{\nu-1}^{m}(x)-(\nu+m-1) R_{\nu-2}^{m}(x)
$$

where we have written $\nu=n-\frac{1}{2}$ and $R_{\nu}{ }^{m}(x)$ may be either $P_{\nu}{ }^{m}(x)$ or $Q_{\nu}{ }^{m}(x)$. Eq. (1) holds for any type of Legendre function. The toroidal harmonics are characterized by $x \geqq 1$ and $m$ and $n$ integral. Since $R_{n-\frac{1}{2}}^{m}(x)=R_{-n-\frac{1}{2}}^{m}(x)$, the computations are carried out for $n \geqq 0$ only.

If two key values $P_{\nu}{ }^{m}(x)$ and $P_{v+1}^{m}(x)$ are given, eq. (1) can be used to generate $P_{v+k}^{m}(x)$ with good accuracy since $P_{v+k}^{m}(x)$ increases with increasing $k$. However, the $Q_{\nu}{ }^{m}(x)$ decrease with increasing order and it is necessary to recur in the direction of decreasing order. The method of finding the key values is described in the next section.

2. Method of Calculation. Many formulas exist for the calculation of $P_{y}{ }^{m}(x)$ [2], [3]. One which is well suited for machine computation is

$$
P_{\nu}{ }^{m}(x)=\frac{\Gamma(\nu+m+1)}{m ! \Gamma(\nu-m+1)}\left(\frac{x-1}{x+1}\right)^{m / 2} \frac{F(-\nu, m-\nu, m+1 ;(x-1) /(x+1))}{[(x+1) / 2]^{-\nu}}
$$

where $F(a, b, c ; z)$ is the hypergeometric function which can be computed from the series expansion [3]:

$$
F(a, b, c ; z)=\frac{\Gamma(c)}{\Gamma(a) \Gamma(b)} \sum_{n=0}^{\infty} \frac{\Gamma(a+n) \Gamma(b+n)}{\Gamma(c+n) n !} z^{n} .
$$

Since $a$ and $b$ are half-integral and $c$ is an integer, the calculation is straightforward and the series converges very rapidly for all moderate values of $x$. Since the first term in the series is unity and the series becomes monotonically decreasing after a finite number of terms, the computation continues until the next added term is less than some small value $\epsilon . P_{-1}^{m}$ and ${P_{\frac{1}{2}}}^{m}$ are chosen as the key values and the recurrence relation is used to get functions of higher order.

The calculation of the function of the second kind is somewhat more involved and a method suggested by Goldstein and Thaler [1] is used. $Q_{v+1}^{m}(x)$ is set to zero and $Q_{\nu}{ }^{m}(x)$ to a small value $\delta$. Then the recursion relation is used to get the functions of lower order. It is necessary to begin computing with functions of fairly high order to get good accuracy. It is difficult to give an exact prescription, but for small values of $x$ one should begin with the function of order $2 \nu$ to get order $\nu$ accurately. For values of $x>5$, the functions increase very rapidly with decreasing order and only a few terms greater than $\nu$ are needed.

The values of $Q_{\nu}{ }^{m}(x)$ obtained by recursion are not properly normalized, and normalization can be effected by computing $Q_{-\frac{1}{3}}^{m}(x)$ say, from the formula [2]:

$$
\begin{aligned}
Q_{\nu}{ }^{m}(x)=\frac{\sqrt{\pi} \Gamma(\nu+m+1)}{2^{\nu+1}\left(\nu+\frac{1}{2}\right) !} & \left(\frac{x^{2}}{x^{2}-1}\right)^{m / 2} \frac{1}{x^{\nu+1}} \\
& F\left(\frac{\nu-m+1}{2}, \frac{\nu-m+2}{2}, \frac{2 \nu+3}{2} ; \frac{1}{x^{2}}\right)
\end{aligned}
$$

and normalizing the other computed functions to this value. For this normalization all values of $Q_{\nu}{ }^{m}(x)$ are positive. Many authors include a factor $(-1)^{m}$ on the right 
side of eq. (4). The hypergeometric function appearing in eq. (4) can be computed as described above but convergence is slow for values of $x$ close to unity, and in this case it may be desirable to compute the functions differently [4].

A subroutine [5] has been written for the IBM 704 to calculate the toroidal harmonics using the methods described here. For given $m$ and $x$ a table is obtained of the first twenty values of $P_{n-1}^{m}(x)$ and $Q_{n-1}^{m}(x)$ i.e. $n=0,1, \cdots, 19$ as well as their derivatives using the formula [2]:

$$
\left(x^{2}-1\right) \frac{d}{d x} R_{n-3}^{m}(x)=\left(n-m+\frac{1}{2}\right) R_{n+3}^{m}(x)-x\left(n+\frac{1}{2}\right) R_{n-\frac{1}{3}}^{m}(x)
$$

Because the functions increase very rapidly with both $m$ and $x$, it is convenient to make the restriction $x<40, m \leqq 21$. Where tabulated values exist [2], the code is found to give full agreement. In some other cases, the equation for the Wronskian [3] of the solutions was checked and found to be very accurately satisfied. The code computes correctly the toroidal harmonics to at least six significant figures.

Atomic Energy Commission

Computing and Applied Mathematics Center

New York University

New York, New York

1. M. Goldstein \& R. M. Thaler, "Calculation of Bessel functions," MTAC, v. 13, 1959, p. 102-108.

2. NBS Mathematical Tables Project, Tables of the Associated Legendre Function, Columbia University Press, New York, 1945.

3. P. M. Morse \& H. FeshBach, Methods of Theoretical Physics, McGraw-Hill Book Company, New York, 1953.

4. J. P. AUFFraY (private communication).

5. A. Rotenberg, NU ASLG, Share Distribution 711, Share Program Librarian, IBM, 590 Madison Avenue, New York 22, New York.

\section{Transcendental Equation tor the Schrödinger Equation}

\section{By J. R. M. Radok}

The problem of the determination of the energy levels of single particles in cylindrical wells of different dimensions reduces to the transcendental equation

$$
\mathfrak{h}_{\lambda}{ }^{(1)}\left(i-\sqrt{k^{2}-\epsilon^{2}}\right)+\mathfrak{h}_{\lambda}(\epsilon)=0
$$

for the Schrödinger equation, where $\mathfrak{h}_{\lambda}{ }^{(1)}, \mathfrak{y}_{\lambda}$ are modified quotient Bessel functions for which a table has been published recently by Morio Onoe [1] and the variables

$$
k^{2}=\frac{2 m U a^{2}}{\hbar^{2}}, \quad \epsilon^{2}=2 m \frac{(E+U) a^{2}}{\hbar^{2}}
$$

involve the quantities

$$
\begin{aligned}
m & =\text { mass } \\
U & =\text { potential energy, }
\end{aligned}
$$

Received January 16, 1959; in revised form, January 18, 1960. 